\title{
Ultrasound-guided vascular access: the importance of the needle bevel
}

\author{
Geza Reusz, MD • Csilla Langer, MD • \\ Lajos Jakab, MD $\cdot$ Zita Morvay, MD, PhD
}

Received: 24 January 2012/ Accepted: 15 February 2012/Published online: 7 March 2012

(C) Canadian Anesthesiologists' Society 2012

\section{To the Editor,}

Real-time ultrasound guidance has become a standard of care for the placement of central venous catheters, and it is gaining in popularity for other types of vascular access, such as cannulation of arteries and peripheral veins. ${ }^{1}$ An "out-of-plane" or "in-plane" technique can be utilized (the path of the needle is directed perpendicular or parallel to the ultrasound beam, respectively); however, with either technique, correct identification of the needle tip during advancement is crucial to avoid complications. The in-plane approach, although technically more challenging, offers the advantage of visualizing the entire length of the needle, thereby possibly reducing posterior vessel wall perforations and punctures of neighbouring organs. ${ }^{2}$

We used a LOGIQ Book XP Pro ultrasound machine (GE Medical Systems Co., Ltd., Wuxi, China) and a $10 \mathrm{MHz}$ linear probe and 20G over-the-needle cannula (Becton Dickinson Critical Care Systems Pte Ltd., Singapore) to perform an ultrasound-guided radial artery cannulation using an in-plane approach. During the

Electronic supplementary material The online version of this article (doi:10.1007/s12630-012-9683-y) contains supplementary material, which is available to authorized users.

G. Reusz, MD $(\bowtie) \cdot$ C. Langer, MD

Department of Anaesthesia and Intensive Care,

Markhot Ferenc Hospital, Eger, Hungary

e-mail: reuszgeza@gmail.com

L. Jakab, MD

Department of Radiology, Markhot Ferenc Hospital, Eger, Hungary

Z. Morvay, MD, PhD

Department of Radiology, Faculty of Medicine,

University of Szeged, Szeged, Hungary procedure, we observed a short bright line in front of and below the apparent needle tip which was consistently moving along with the needle. Interestingly, now that we look for it, we always see a similar bayonet-like line on the image. This pattern is clearer when the needle tip is intravascular and not obscured by tissues and when the introducer needle (bevel up) is in perfect alignment with the plane of the ultrasound beam (Figure A). Provided a high-frequency probe is used, the line is recognizable with any standard long-bevel intravascular needle or cannula under various ultrasound settings.

Artifacts are commonly seen with ultrasound imaging, ${ }^{3}$ and although this short line might be regarded as an insignificant artifact, we regard this as a real image that must be interpreted correctly. The short line corresponds to the posterior wall of the needle and could be referred to as the "bevel line". High-resolution ultrasound can distinguish between two points separated by the distance between the anterior and posterior walls of the needle shaft, but the acoustic shadow of the highly reflective anterior needle wall usually obscures the view of the posterior wall. However, when the needle is oriented with the bevel facing upwards, the posterior wall of the needle becomes visible close to the needle tip, past the end of the anterior wall (Figure B). Despite a thorough MEDLINE® search, we did not find a publication about the ultrasonic appearance of the needle tip. The concept can be demonstrated easily by inserting the needle into a chicken breast and following its ultrasound image as it is rotated $180^{\circ}$ along its long axis. When the bevel faces up, the broken line corresponding to the posterior wall is clearly seen, but the line disappears when the bevel faces the opposite direction (Video, accessible as supplementary electronic material).

In our view, these observations have two practical consequences. First, although the in-plane approach allows 

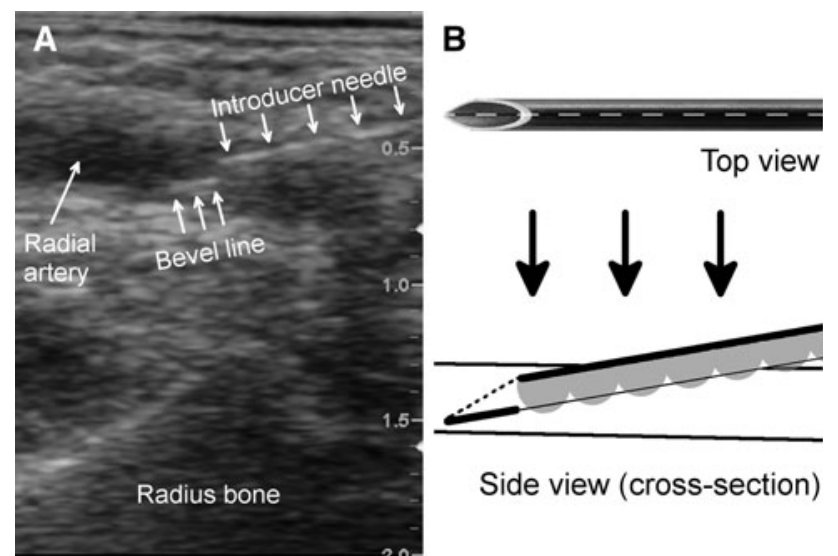

Top view

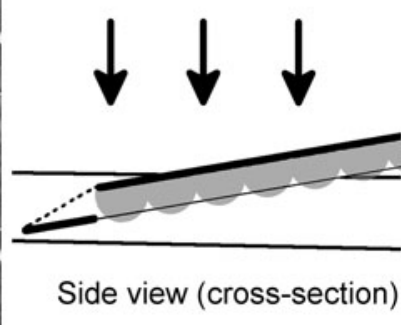

Figure Cannulation of the radial artery (real-time ultrasound guidance, in-plane technique). The tip of the introducing needle appears to be in the middle of the artery (end of long line), but there is another short line in front of and slightly below it (Figure A). The short line, about $2 \mathrm{~mm}$ long, corresponds to the posterior wall of the needle at the bevel where it comes out of the acoustic shadow of the anterior wall (Figure B). The real needle tip is at the distal end of the short line; observe how close it is to the posterior wall of the artery

for a better visualization of the entire needle than the outof-plane technique, the needle tip may actually be more distal than the end of the line representing the image of the needle. We find that the "bevel line" is a useful direct sign that should be actively sought to confirm needle tip position during needle advancement. Second, the actual needle tip is at the distal end of this short bevel line, a few millimetres deeper than the end of the long line representing the apparent needle. This is extremely important when cannulating small-diameter vessels, such as peripheral veins or the radial artery, with the in-plane approach. Neglect of the "bevel line" can lead to inadvertent perforation of the posterior wall of the vessel, which might result in cannulation failure and hematoma formation.

\section{Competing interests None declared.}

No funding was received from any agency or institution.

There are no commercial or non-commercial affiliations, or other associations, that are or may be perceived to be a conflict of interest with the work of any author.

\section{References}

1. Troianos CA, Hartman GS, Glas KE, et al. Special article: Guidelines for performing ultrasound guided vascular cannulation: recommendations of the American Society of Echocardiography and the Society of Cardiovascular Anesthesiologists. Anesth Analg 2012; 114: 46-72.

2. Blaivas $M$, Adhikari $S$. An unseen danger: frequency of posterior vessel wall penetration by needles during attempts to place internal jugular vein central catheters using ultrasound guidance. Crit Care Med 2009; 37: 2345-9.

3. Sites $B D$, Brull $R$, Chan $V W$, et al. Artifacts and pitfall errors associated with ultrasound-guided regional anesthesia. Part II: a pictorial approach to understanding and avoidance. Reg Anesth Pain Med 2010; 35(2 Suppl): S81-92. 